\title{
Study of the dependence of the large scale anisotropy on the nature of the primary cosmic rays with the ARGO-YBJ experiment
}

\author{
Wei Gao* \\ Key Laboratory of Particle Astrophysics, Institute of High Energy Physics, CAS, Beijing 100049, \\ China. \\ University of Chinese Academy of Sciences. 100049 Beijing, China. \\ E-mail: gaoweidihep.ac.cn

\section{Songzhan Chen ${ }^{\dagger}$, Huihai He} \\ Key Laboratory of Particle Astrophysics, Institute of High Energy Physics, CAS, Beijing 100049, \\ China.
}

\section{Shuwang Cui}

Hebei Normal University. 050016 Shijiazhuang , Hebei, China.

\section{On behalf of the ARGO-YBJ Collaboration}

\begin{abstract}
The cosmic ray large scale sidereal anisotropy with an amplitude of the order of $10^{-3}$ has been firmly detected by a number of experiments from sub-TeV to $\mathrm{PeV}$ energies. The large scale anisotropy pattern is quite energy dependent. The amplitude increases with energy, reaches the maximum around $10 \mathrm{TeV}$, while the angular phase is approximately stable at energies below 100 $\mathrm{TeV}$. Different models have been proposed to explain the origin of the anisotropy, concerning different aspects of cosmic ray physics, from the sources of cosmic rays to the propagation to the Earth. Some models consider the anisotropy due to the spatial distribution of cosmic ray sources, as the presence of a nearby strong source, while other interpretations concern the structure of the Galactic and interplanetary magnetic fields. No matter which model, the magnetic field must play an important role, and this would produce differences in the anisotropy among the cosmic ray elements. Therefore, a measurement of the anisotropy dependence on the nature of the primaries could provide important clues to test these models. In this work, we use the ARGO-YBJ data collected from 2008 to 2009 to carry out this study. Data have been selected to obtain two samples of air showers generated by cosmic rays with different elemental composition. Then the models are tested by comparing the anisotropy of the two data samples. The implications on the anisotropy origin are also discussed.
\end{abstract}

35th International Cosmic Ray Conference - ICRC2107

10-20 July, 2017

Bexco, Busan, Korea

${ }^{*}$ This work is supported in China by NSFC No.11575203, No.11375052

† Speaker. 


\section{Introduction}

The arrival directions of cosmic rays are nearly isotropic due to deflections in the Galactic magnetic field. In the past decades, the directional variation of the cosmic ray flux, referred to as anisotropy, with the order of $10^{-3}$ was observed by a number of air shower experiments, such as

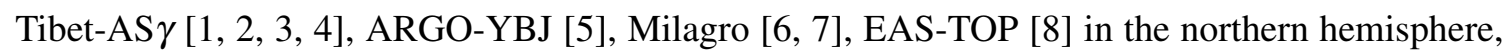
and IceCube/IceTop [Q, [0] in the southern hemisphere. A sidereal large scale anisotropy (LSA) has been detected above $100 \mathrm{GeV}$ with a relative amplitude of the order $10^{-4} \sim 10^{-3}$ as shown in Figure ‥ Below $100 \mathrm{TeV}$, two large stable features known as "tail-in" and "loss-cone" are observed with very high significance [ [3, $[\mathbf{W}]$. The amplitude increases slowly with energy, reaching a maximum at about $10 \mathrm{TeV}$, then decreasing up to $100 \mathrm{TeV}$. Above $100 \mathrm{TeV}$, the anisotropy amplitude increases

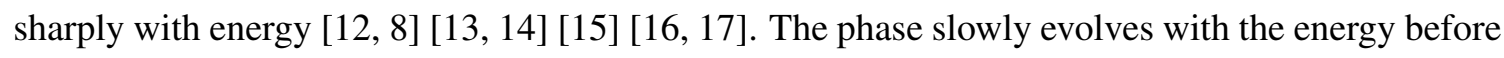
abruptly changing at about $300 \mathrm{TeV}$. The anisotropy evolution at high energies up to $1 \mathrm{PeV}$ has been recently reported by Tibet-AS $\gamma$ [ㅍ] ]. A new component of the the excess around $270^{\circ}$ in right ascension starts appearing at $100 \mathrm{TeV}$ and a deficit at $50^{\circ} \sim 100^{\circ}$ is observed at energies above $300 \mathrm{TeV}$, in agreement with the IceCube results at $400 \mathrm{TeV}$ [[13]. The amplitude and phase variation with energy rule out the Compton-Getting effect [ए8] as the main source of anisotropy. For the solar anisotropy, both Tibet-AS $\gamma$ [B] ] and ARGO-YBJ [U]] observed a dipole anisotropy in fair agreement with the expected Compton-Getting effect as a result of the Earth orbital motion around the Sun. In our paper, we mainly consider the sidereal LSA.

Up to now, the sources of sidereal LSA are still uncertain. Different models have been proposed to explain the origin of the anisotropy, concerning different aspects of cosmic ray physic$\mathrm{s}$, from the sources of cosmic rays to the propagation to the Earth. Some models consider the anisotropy due to the stochastic spatial distribution of supernova explosions, as the presence of a nearby strong source [10]. Other interpretations concern the effects of the magnetic fields, including the global Galactic magnetic field [20], the local interstellar magnetic field surrounding the solar system [제], the magnetic field within heliosphere [22]. No matter in which model, the magnetic fields are expected to play an active role, and this would produce differences in the anisotropy among the cosmic ray elements. Therefore, a measurement of the anisotropy of different mass cosmic rays could provide important clues to test current models.

ARGO-YBJ has been a particle array operating at high altitude. Its unique features (high altitude location, 2 sr field-of-view, full coverage layout, high granularity readout, duty cycle $>$ $85 \%$ ) made it well suitable for investigating the dependence of the anisotropy below $100 \mathrm{TeV}$ on the nature of the primary cosmic rays. The main sources of the cosmic ray anisotropy are introduced in Section 2. The experiment, data selection and reconstruction are presented in Section 3. In Section 4 we report on the measured cosmic ray anisotropy, the analysis of data samples with different element composition and the related implications. The results are summarized and discussed in the last Section.

\section{Sources of the large scale anisotropy}

The origin of the cosmic ray anisotropy remains substantially unknown. The LSA may be qualitatively explained by a standard diffusive propagation of cosmic rays in the Galaxy from stochas- 
tically distributed sources. The amplitude of anisotropy is determined by diffusion coefficients and spatial gradients as $A=\frac{3 D(R) \nabla I}{c I}$ [ए9], here $A$ is the amplitude of the anisotropic component of the cosmic ray distribution, $R=E / Z$ is the cosmic ray rigidity, $E$ is the cosmic ray energy, $Z$ is the nuclear charge of the cosmic ray particle, $I$ is the cosmic ray intensity, $D(R) \sim R^{\delta(0.3-0.6)}$ is the diffusion coefficient. For individual elements, at a given energy the heavy particle with bigger $Z$ has smaller diffusion coefficient, which lead to a weaker amplitude. Thus the amplitude of the anisotropy is expected to be related to the cosmic ray composition. In the interstellar magnetic field, the parallel component of the diffusion is dominating in the transfer process, and the cosmicray gradient in this case depends largely on the ratio of perpendicular to parallel diffusion [지]. In addition, cosmic ray sources such as nearby strong sources can also affect the cosmic ray density gradient [미 so that impact on the anisotropy as well.

When charged particles travel in the local interstellar magnetic field through the Galaxy, the gyromagnetic radius at a given energy depends on the particle charge $r_{\text {gyro }}=1 \mathrm{kpc} \frac{1}{Z} \frac{E}{10^{8} \mathrm{eV}} \frac{\mu G}{B}$. Heavy particles with high $Z$ are more easily deflected than light particles. Thus a cosmic ray mixture rich of heavy nuclei is more easily diffused, leading to an anisotropy weaker than that of a cosmic ray sample rich in light elements. The greater anisotropy of light elements should be enhanced if they come from nearby sources. In another situation, when isotropic cosmic rays travel in the vicinity environment, the magnetic field inflects their directions. Heavy particles are more easily driven along the magnetic field lines, inducing an anisotropic distribution. Thus the amplitude has a positive relation with the particle charge $(Z)$. If the anisotropy is produced by the magnetic field, then cosmic ray samples rich in heavy nuclei will have larger anisotropy amplitude. Therefore the study of the anisotropy of different primary nuclei could help to better clarify its origin.

The Compton-Getting (CG) effect [ए]] is also considered as a possible anisotropy source. Assuming that the cosmic rays are isotropic in the Galaxy, the rotation of the solar system about the Galactic Centre should lead to a dipole anisotropy with amplitude of about $\sim 10^{-3}$ according to the CG effect. As shown in Figure $\square$, the amplitude is observed to increase with energy up to about $10 \mathrm{TeV}$ before flattening while the phase smoothly evolves before undergoing a sudden flip at about $300 \mathrm{TeV}$ [ए]7, [4]]. The observed characteristics of the cosmic ray anisotropy are inconsistent with a simple dipole model. Hence, we can rule out the CG effect as the dominant source of anisotropy.

\section{Data selection and energy reconstruction}

\subsection{The ARGO-YBJ experiment}

The ARGO-YBJ experiment has been a full coverage air shower array located in YangBaJing, Tibet, China (4300 $\mathrm{m}$ above the sea level). It consisted of a centre full coverage carpet $\left(74 \times 78 \mathrm{~m}^{2}\right.$, 130 clusters) and a guard ring (23 clusters). Each cluster $\left(5.7 \times 7.6 \mathrm{~m}^{2}\right.$ each) is composed of 12 resistive plate chambers (RPC, $1.23 \times 2.85 \mathrm{~m}^{2}$ each). Each RPC is read out by 10 pads $(55.6 \times$ $61.8 \mathrm{~cm}^{2}$ each), representing the spacetime pixels of the detector. Each pad is composed of 8 strips. The detector took data from November 2007 to February 2013 with an inclusive trigger $N_{\text {pad }}>20$, being $N_{\text {pad }}$ the number of fired pads in the central carpet. The trigger rate was about $3.5 \mathrm{kHz}$, accidental free. With this trigger the energy threshold is a few hundred GeV. For the current analysis the showers are selected according to the following criteria: (1) more than 40 fired 
strips in the central carpet $N_{s t r} \geq 40$, (2) reconstructed zenith angle less than $50^{\circ}$, (3) reconstructed core location less than $100 \mathrm{~m}$ from the carpet centre. About $1.0 \times 10^{11}$ events survive these cuts.

\subsection{Energy reconstruction}

The cosmic ray energy has been estimated by a Monte Carlo (MC) simulation. The interaction of cosmic rays in the atmosphere was simulated by CORSIKA, with the hadronic interaction model QGSJET-II. The detector response was simulated with G4argo [24]. The composition and spectrum models of primary cosmic rays are given in [25]. In this work, we have generated five groups of dominant components of cosmic rays $(P, \mathrm{He}, \mathrm{CNO}, \mathrm{MgSi}, \mathrm{Fe})$. About $4 \times 10^{10}$ events are sampled in the zenith angle band from $0^{\circ}$ to $60^{\circ}$ in the energy range $0.1 \mathrm{TeV} \sim 10 \mathrm{PeV}$.

Generally, the number of fired strips $N_{s t r}$ is solely adopted to infer the primary energy of an air shower. However, the air showers reaching the detectors with a large zenith angle $\theta$ travel through deeper distance in atmosphere than the vertical ones. For the events with same $N_{s t r}$, large zenith angle events have higher primary energy than the little zenith angle events. Therefore the estimate of the primary energy of cosmic rays must consider the dependence on the zenith angle. Figure $\square$ shows the dependence of the primary energy on the strip number $N_{s t r}$ (left plot) and on the zenith angle (middle plot). The right plot of Figure $\mathbb{0}$ gives the obtained linear relation (in a log-log plot) between the estimated shower energy and the primary energy.
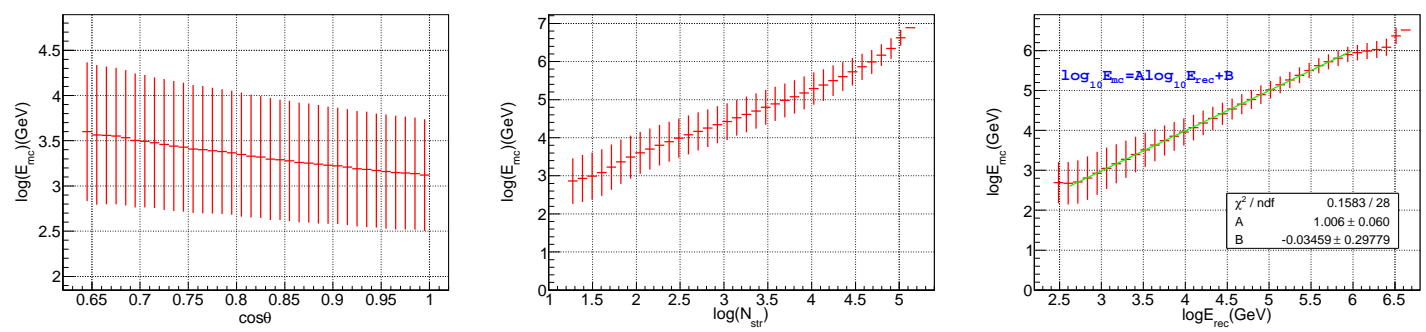

Figure 1: Energy reconstruction. The simulated distribution of the log-energy (in units of GeV) of primary CRs $\left(E_{m c}\right)$ as a function of the zenith angle (left plot) and of $N_{s t r}$ (middle plot). The reconstructed energy of primary cosmic rays is shown in the right plot.

\section{Results}

\subsection{Energy dependence}

The study of the dependence of the TeV large scale anisotropy on the cosmic ray elemental composition is based on the data collected in the 2008-2009 years. The events are selected according to the prescriptions given in Section 2. The showers are divided in seven samples of the reconstructed energy as listed in Table $\mathbb{W}$. The analysis follows the procedure given in [W]. The projection of the intensity $I$ on the right ascension (1D profile) is fitted by the first harmonic function $I=1+a m p \times \cos (\alpha-\phi)$, where $a m p$ is the amplitude of the first harmonic and $\phi$ the phase at which $I$ reaches its maximum. The results (red squares) are plotted in Figure $\square$. These data extend beyond $30 \mathrm{TeV}$ the previous results of ARGO-YBJ given in [W]. The agreement with the previous analysis is excellent, while the result at the highest energy, $185 \mathrm{TeV}$, is suggesting an increasing 


\begin{tabular}{c|c|c|c|c|c}
\hline \hline$N$ & $\log _{10} E_{r e c}(\mathrm{GeV})$ & $E_{m}(\mathrm{TeV})$ & $N U M\left(\times 10^{8}\right)$ & $a m p$ & $\phi\left(^{\circ}\right)$ \\
\hline 1 & $3.50 \sim 3.75$ & 4.0 & 71 & $9.66 \times 10^{-4} \pm 0.17 \times 10^{-4}$ & $29.73 \pm 1.00$ \\
\hline 2 & $3.75 \sim 4.00$ & 7.0 & 38 & $12.33 \times 10^{-4} \pm 0.23 \times 10^{-4}$ & $32.55 \pm 1.07$ \\
\hline 3 & $4.00 \sim 4.25$ & 12.0 & 20 & $11.55 \times 10^{-4} \pm 0.31 \times 10^{-4}$ & $31.37 \pm 1.55$ \\
\hline 4 & $4.25 \sim 4.50$ & 21.0 & 10 & $10.67 \times 10^{-4} \pm 0.44 \times 10^{-4}$ & $27.19 \pm 2.35$ \\
\hline 5 & $4.50 \sim 4.75$ & 39 & 5.0 & $9.16 \times 10^{-4} \pm 0.64 \times 10^{-4}$ & $0.40 \pm 3.97$ \\
\hline 6 & $4.75 \sim 5.00$ & 70 & 2.2 & $5.91 \times 10^{-4} \pm 0.96 \times 10^{-4}$ & $354.03 \pm 9.28$ \\
\hline 7 & $\geq 5.00$ & 185 & 1.5 & $6.91 \times 10^{-4} \pm 1.16 \times 10^{-4}$ & $231.09 \pm 9.64$ \\
\hline \hline
\end{tabular}

Table 1: The median energy, events number, amplitude and phase of seven energy samples. The amplitude and phase are get from the best fit of the 1D profile.

amplitude above $100 \mathrm{TeV}$, consistent with the results obtained by other experiments (see Figure [2). The two-dimensional map in equatorial coordinates (2D) is reported in Figure B] (a smoothing with a window width of $30^{\circ}$ has been applied), and the one-dimensional (1D) projection of the relative intensity is shown in Figure 6 . The pre-trial significance of the deficit is about $-5.09 \sigma$

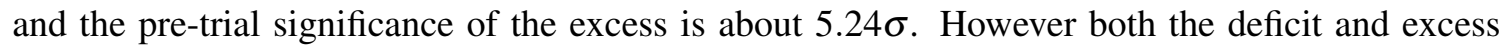
regions are consistent with the results of $\mathrm{AS} \gamma$ at 300-1000 TeV [ए]] and of IceCube at $400 \mathrm{TeV}$ in the southern hemisphere [ㅍ]]. Thus it is confirmed that the anisotropy map at energies greater than $100 \mathrm{TeV}$ is different from that at multi-TeV energies characterized by the so-called tail-in and loss-cone features.
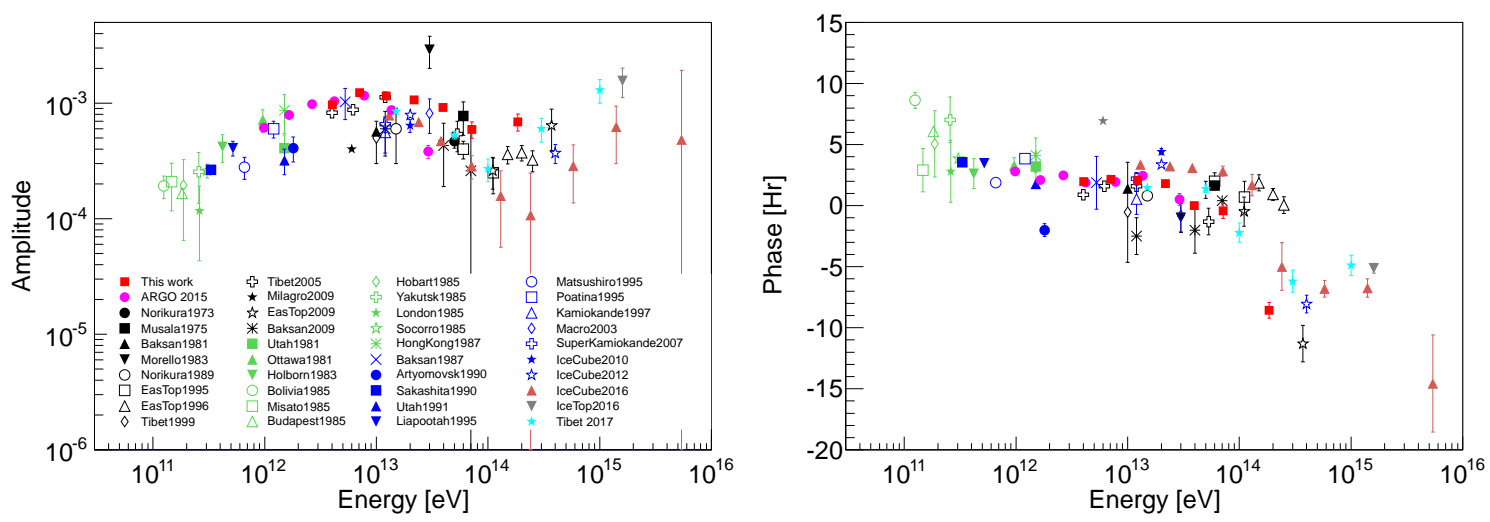

Figure 2: The amplitude (left plot) and the phase (right plot) of the first harmonic of the sidereal anisotropy measured by ARGO-YBJ (red squares), as a function of the cosmic ray energy (in the units of eV), along with the results from other experiments.

\subsection{Dependence on the cosmic ray elemental composition}

A preliminary study of the sidereal anisotropy dependence on the cosmic ray elemental composition has been carried out on the data sample collected at a median energy of $10 \mathrm{TeV}$ (see Table Ш). The events have been grouped in two samples according to the strip distribution around the core. The expected composition of these samples, as obtained by a MC simulation, is reported in Table ㅁ. The $\mathrm{v} 1$ sample appears richer in light elements with respect to the v2 sample. Figure $[$ shows 

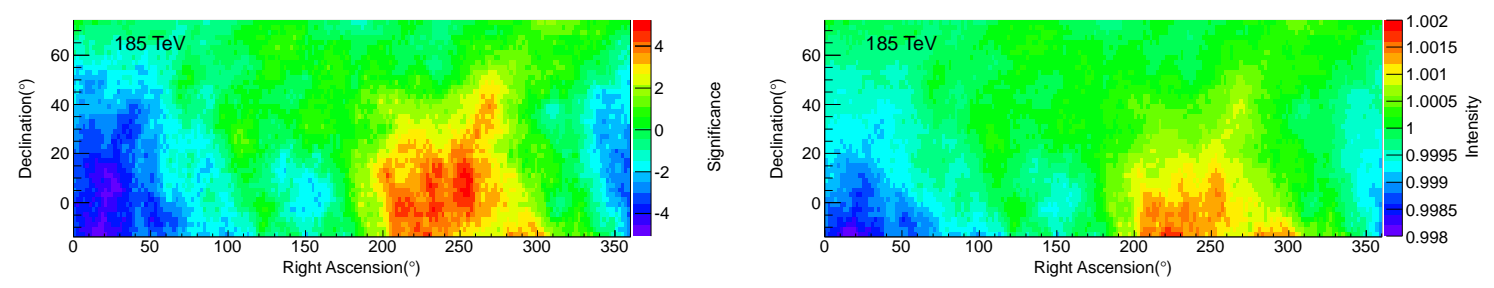

Figure 3: 2D anisotropy maps (with $30^{\circ}$ smoothing) at the median energy of $185 \mathrm{TeV}$ (left plot: significance, right plot: relative intensity).

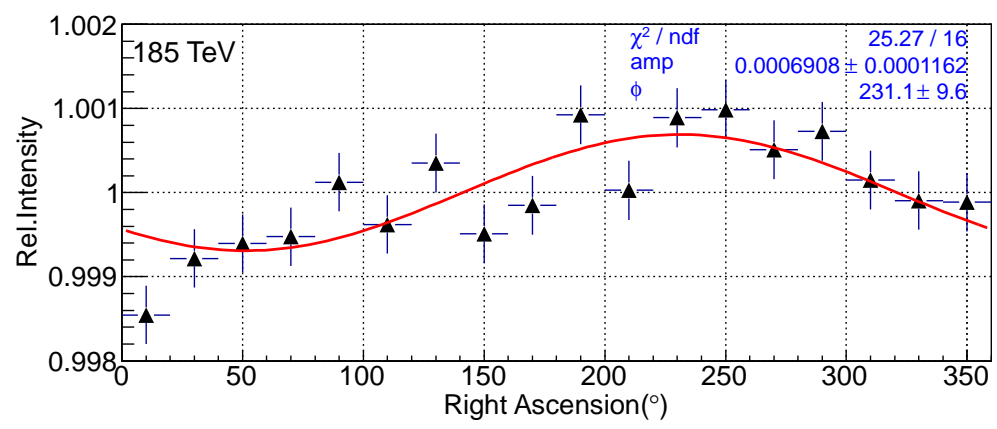

Figure 4: $1 \mathrm{D}$ projection of the relative intensity at the median energy of $185 \mathrm{TeV}$.

\begin{tabular}{c|c|c|c|c|c|c|c|c}
\hline \hline & $\mathrm{P}$ & $\mathrm{He}$ & $\mathrm{CNO}$ & $\mathrm{MgAlSi}$ & $\mathrm{Fe}$ & $\mathrm{NUM}\left(\times 10^{8}\right)$ & $\mathrm{amp}\left(\times 10^{-4}\right)$ & $\phi\left(^{\circ}\right)$ \\
\hline $\mathrm{v} 1$ & $82.9 \%$ & $16.5 \%$ & $0.6 \%$ & $0.1 \%$ & 0.0 & 3.41 & $14.23 \pm 0.77$ & $43.02 \pm 3.08$ \\
\hline $\mathrm{v} 2$ & $66.1 \%$ & $27.6 \%$ & $4.2 \%$ & $1.1 \%$ & $1.0 \%$ & 68.6 & $11.85 \pm 0.17$ & $31.30 \pm 0.83$ \\
\hline \hline
\end{tabular}

Table 2: Composition, anisotropy amplitude and phase for the two samples v1 and v2.

the $2 \mathrm{D}$ maps, with $15^{\circ}$ smoothing, of these samples. The $1 \mathrm{D}$ projection of the relative intensity for both samples is shown in Figure (left plot). The anisotropy pattern of the two samples looks quite similar, with only a slight difference, at a level of $3.8 \sigma$ significance, in the amplitude of the deficit and excess regions (Figure 6 , right plot).

Assuming $A_{p}$ as the anisotropy amplitude of the proton component, we model the amplitude of heavier nuclei according to the relation (4.])

$$
A(Z)=A_{p} \times Z^{\beta}
$$

Thus the expected anisotropy amplitude $\langle A\rangle$ of each sample is given by the relation (4.2)

$$
\langle A\rangle=A_{p} \times \sum\left[\xi(Z) \times Z^{\beta}\right]
$$

where $\xi(Z)$ is the weight of the element of charge $Z$ in the sample. A global fit to data gives $\beta=-2.29 \pm 1.95$. The large error on beta prevents us to reach any firm conclusion about the anisotropy of heavy nuclei. Taken the beta estimate at its face value, this result could suggest that the light elements, as protons and helium nuclei, are considerably more anisotropic than the heavy nuclei, a picture consistent with the presence of strong nearby sources. 

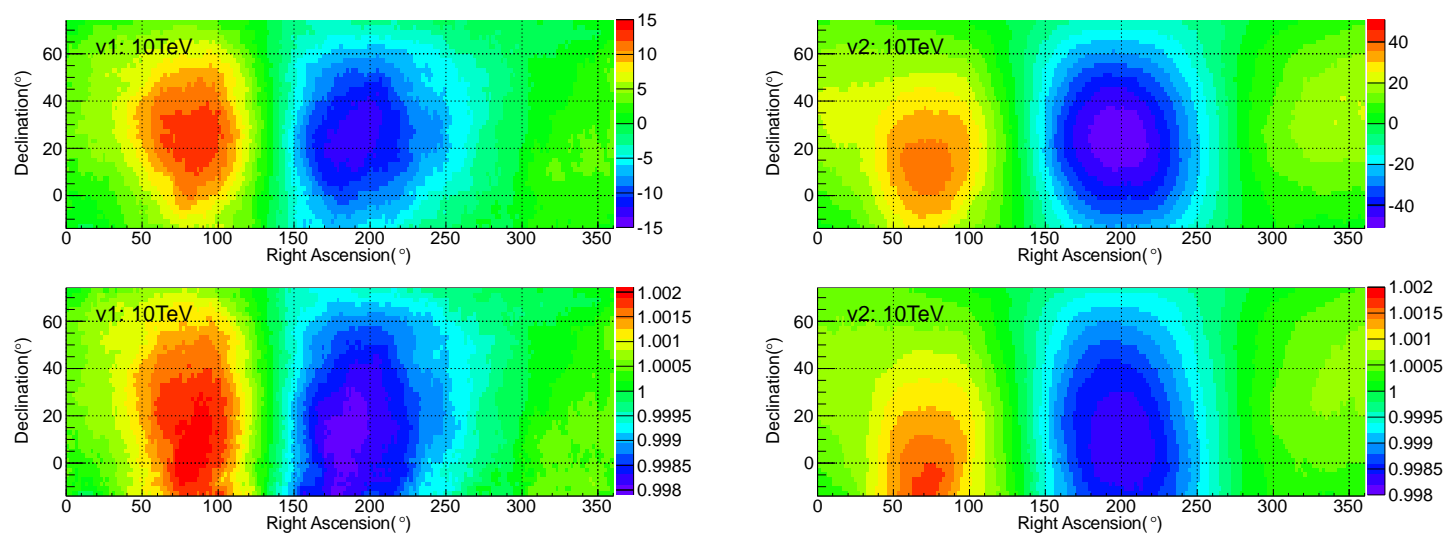

Figure 5: The relative intensity and the significance 2D maps smoothed with $15^{\circ}$ radius for sample v1 (left) and $\mathrm{v} 2$ (right).
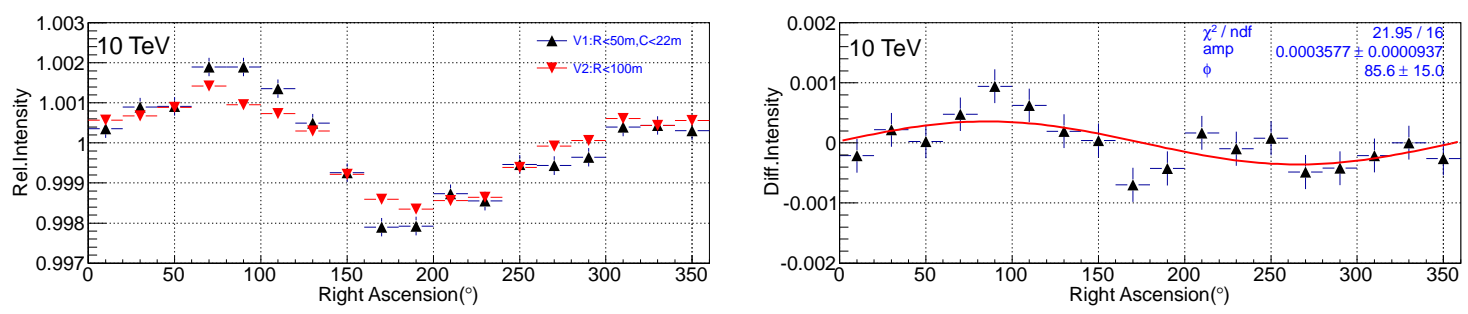

Figure 6: The 1D projections of the relative intensity for the v1 and v2 samples (left plot). The intensity difference of the $\mathrm{v} 1$ and $\mathrm{v} 2$ samples(right plot).

\section{Summary}

This paper reports on the measurement of the large scale cosmic ray anisotropy by the ARGOYBJ experiment with the data collected from 2008 to 2009. In this work, we improve the estimate of the primary cosmic ray energy by including the larger zenith angle events and considering the appropriate correction in the energy reconstruction. Comparing with our previous work, the energy range has been expanded from $30 \mathrm{TeV}$ to $185 \mathrm{TeV}$. At low energies the 2D anisotropy maps and the amplitude and phase of the 1D profile are in good agreement with the previous results. At $185 \mathrm{TeV}$, the 2D anisotropy map shows a very different pattern with respect to the ones found in the multi-TeV range. Fitting the 1D profile by a first harmonic function we find a deficit region centered at about $\alpha=70^{\circ}$ and an excess region centered at about $\alpha=240^{\circ}$, values consistent with the ones found by As $\gamma$ and IceCube at high energies. The variation of the $2 \mathrm{D}$ anisotropy map at high energies is clearly indicating a new component of the anisotropy. This effect could be correlated with the energy evolution of the primary cosmic ray composition. A preliminary study of the cosmic ray anisotropy dependence on the elemental composition has been carried out using showers at $10 \mathrm{TeV}$ median energy .

The events have been subdivided in two samples with different mixture of nuclei. Since the diffusion process depends on the particle rigidity, we have assumed that, at a given energy, the anisotropy amplitude of the nuclei with charge $Z$ is related to $Z$ as $Z^{\beta}$. The best estimate of beta that reproduces the observed anisotropies is then obtained, $\beta=-2.29 \pm 1.95$. Even if affected by 
a large uncertainty, this beta estimate favours a larger anisotropy of the light elements, as protons and helium nuclei, than that of the heavy nuclei. As a very preliminary guess, we can envisage the origin of these cosmic rays mainly from nearby sources. We plan to increase the statistics including events collected in other years, to improve the composition selection and to extend this study to higher energies.

\section{References}

[1] M. Amenomori et al. 2005, ApJ, 633,1005

[2] M. Amenomori et al. 2005, ApJ, 626,L29

[3] M. Amenomori et al. 2006, Science, 314

[4] M. Amenomori et al. 2010, ApJ, 711,119

[5] J. L. Zhang et al. 2009, in Proc. 31st ICRC

[6] A. A. Abdo et al. 2008, PRL, 101,221101

[7] A. A. Abdo et al. 2009, ApJ, 698,2121

[8] M. Aglietta et al. 2009, ApJ, 692,L130

[9] R. Abbasi et al. 2010, ApJL 718,L194

[10] R. Abbasi et al. 2011, ApJ, 714,L194

[11] B. Bartoli et al. 2015, ApJ, 809,90

[12] M. Aglietta et al. 1996, ApJ, 470,501

[13] R. Abbasi et al. 2012, ApJ, 746,33

[14] M. G. Aartsen et al. 2016, ApJ, 826,220

[15] M. G. Aartsen et al. 2013, ApJ, 765,55

[16] M. Amenomori et al. 2013, in Rio. 33rd ICRC, ID256

[17] M. Amenomori et al. 2017, ApJ, 836,153

[18] A. H.Compton, I. A. Getting 1935, PRL, 47,817

[19] A. D. Erlykin, A. W. Wolfendale 2006, Astropart.Phys., 25,183

[20] X. B. Qu et al. 2012, ApJL, 750

[21] N. A. Schwadron et al. 2014, Science, 343,988

[22] M. Zhang et al. 2014, ApJ, 790, 5

[23] B. Bartoli et al. 2012, PRD,85,092005

[24] Y. Q. Guo te al. 2010, ChPhC, 34,555

[25] T. K. Gaisser et al. 2013, Front.Phys,8(6),748 